The phenomenon of standing waves of light in the form of the Lippmann process was the subject of Prof. Ives's doctoral thesis some forty years ago. He then turned, during the First World War, to work on aerial photography, and after the War at the Bell Telephone Laboratories he was occupied at first with electrical contacts and then with the photoelectric effect, of which he made a close study. The various problems in the nature of the photoelectric effect, particularly the effect of the state of polarization of the exciting light on the photoelectric emission and the spectral distribution of the photoelectric emission from the alkalis, occupied his attention and led him to give an explanation in terms of the existence of a system of standing waves above the metal surfaces. This required for its test a knowledge of the optical constants of the alkali metals. Details of the experimental results obtained and their significance are discussed briefly by Prof. Ives. He stresses that it is the optical properties of the materials and the optical conditions of the region in which the phenomena occur which explain the outstanding effects, and that the various quantum mechanical explanations have almost uniformly neglected or ignored the optical factors.

Another experiment with standing waves of light referred to by Prof. Ives is a modification of Wiener's experiment, with a photoelectric film replacing the photographic film used by Wiener. Prof. Ives asserts that the verification of the electromagnetic wave theory of light furnished by this experiment considerably transcends that of Wiener's original simple experiment.

Finally, Prof. Ives discusses what contribution the results of these photoelectric experiments can make to the elucidation of the old question of the nature of light, waves versus corpuscles. Wiener's modified experiment, he claims, comes very close to showing both types of phenomena in combination. Wave mechanics is a way out of the dilemma. It avoids the unacceptable idea of photons by putting the quantum $h$ in the atom and not in the radiation, and Prof. Ives concludes by predicting that the photon will go the way of the 'calorie' that Rumford demolished.

\section{X-RAY AND NEUTRON MUTAGENIC EFFECTS ON CEREAL SEEDS}

$\mathrm{T}$ HE biological action of X-rays and fast neutrons on cereal seeds is the subject of detailed investigation in the Mutation Laboratory of the Swedish Seed Association at Svalöf, Sweden, use being made of the cyclotron equipment and other facilities of the Nobel Institute for Physics and of the Institute of Radiophysics at Stockholm (J. Mackey, Arkiv. för Botanik, 1, 16, 545 ; L. Ehrenberg, A. Gustafsson and N. Nybom, ibid., 557, 1952).

Both dry dormant and soaked seeds of wheat and barley were irradiated with X-rays and fast neutrons and the treated materials followed through two generations for observation of the frequency of primary chromosome disturbances, the retarding effect on sprouting, the death-rate, the induced degree of sterility in the first generation, and the frequency of certain types of mutation in the second. For the production of genetically interesting and useful materials, neutrons are many times more effective than X-rays; at high neutron dosages the mutation-rate reaches a very high level. Cells with low water content (and low metabolic activity) are highly resistant to the killing effects of neutrons as compared with X-rays, but actively dividing cells are more sensitive to neutrons ; after neutron irradiation, about five times as many cells show chromosome disturbances in pre-soaked as compared with dry seed.

Different radiations may act in different ways because of differences in ionization density, distribution of ionizing rays within the object, and the duration of the time of action of isotopes. The general result so far obtained is that neutrons are about ten times as effective as X-rays of equivalent energy dissipation in causing chromosome disturbances and from fifty to one hundred times as effective in causing first-generation sterility and in increasing the rate of mutation. This higher effectiveness is attributed to the difference in ion density. While the results reported are not regarded as final, they constitute an important advance in the planned and controlled induction of mutations in agricultural and horticultural plants by ionizing agents.

\section{CYTOCHROME COMPONENTS IN CHLOROPLASTS}

\section{BY H. E. DAVENPORT}

\section{School of Biochemistry, University of Cambridge}

ITTLE was known of the distribution of components of the cytochrome system in green leaves until Hill and Scarisbrick ${ }^{1}$ separated not only cytochrome $c$ but also two new soluble components, $b_{3}$ and $f$. Cytochrome $f$ appeared to be confined to chlorophyll-containing tissues where the presence of high concentrations of plastid pigments precludes the direct spectroscopic observation of the component. It was first observed in the reduced form in preparations of leaves from which plastjd pigments had been removed by acetone treatment. Afterwards, Hill and Scarisbrick obtained cytochrome $f$ in solution in ethanol extracts of fresh leaves. In solution it is not autoxjdizable, does not combine with carbon monoxide and has, in the reduced form, a characteristic spectrum with the $\alpha$-band at $555 \mathrm{~m} \mu$. Further properties of the purified cytochrome have been described in greater detail ${ }^{2}$ and evidence presented for supposing that it may play an important part in the electron transfers occurring in photosynthesis. Hitherto, however, cytochrome $f$ has been observed only in preparations which had been treated with organic solvents, and the possibility therefore remained that it was an artefact arising from some other hæmatin compound as a result of that treatment. It has now been found that the absorption spectrum of cytochrome $f$ can be observed directly in intact plastids prepared from etiolated barley leaves.

Barley seedlings were grown on John Innes seedling compost in a darkened incubator at about $20^{\circ} \mathrm{C}$. After nine days, the yellow leaves were harvested and 10-gm. amounts were ground in a chilled mortar with $10 \mathrm{ml}$. of cold $0.5 \mathrm{M}$ glucose in $0.03 \mathrm{M}$ phosphate buffer of $p H \mathbf{7 \cdot 4}$. The material was squeezed through muslin and centrifuged for I min. at 3,000 r.p.m. to remove intact cells and vascular debris. The turbid yellow supernatant fluid was centrifuged at 4,000 r.p.m. at $2^{\circ}$ C. for a further 5 min. This gave a yellowish brown sediment which when viewed 